\title{
Coupling Single-Drop Microextraction with SERS: A Demonstration Using p-MBA on Gold Nanohole Array Substrate
}

\author{
Elias B. Santos ${ }^{1}$, Chiara Valsecchi ${ }^{2}, * \mathbb{1}$, Jaderson L. S. Gonçalves ${ }^{2}$, Luis F. Ávila ${ }^{3}$ \\ and Jacson W. Menezes ${ }^{2}$ (D) \\ 1 LQANano, Federal University of São Paulo, São José dos Campos-SP, 12231-280, Brazil; \\ santos.barros@unifesp.br \\ 2 Engineering Department, Federal University of Pampa, Alegrete-RS, 97546-550, Brazil; \\ jadersg@alunos.unipampa.edu.br (J.L.S.G.); jacsonmenezes@unipampa.edu.br (J.W.M.) \\ 3 Applied Optics Laboratory-School of Technology, State University of Campinas, \\ Limeira-SP, 13484-350, Brazil; lfavila@ft.unicamp.br \\ * Correspondence: chiaravalsecchi@unipampa.edu.br
}

Received: 10 August 2019; Accepted: 19 September 2019; Published: 11 October 2019

\begin{abstract}
Single-drop microextraction (SDME) was coupled with surface-enhanced Raman scattering (SERS) to provide sample extraction and pre-concentration for detection of analyte at low concentrations. A gold nanohole array substrate (AuNHAS), fabricated by interference lithography, was used as SERS substrate and para-mercaptobenzoic acid (p-MBA) was tested as a probe molecule, in the concentration range $10^{-8}-10^{-4} \mathrm{~mol} \mathrm{~L}-1$. With this approach, a limit of $10^{-7} \mathrm{~mol} \mathrm{~L}^{-1}$ was clearly detected. To improve the detection to lower p-MBA concentration, as $10^{-8} \mathrm{~mol} \mathrm{~L}^{-1}$, the SDME technique was applied. The p-MBA Raman signature was detected in two performed extractions and its new concentration was determined to be $\sim 4.6 \times 10^{-5} \mathrm{~mol} \mathrm{~L}^{-1}$. This work showed that coupling SDME with SERS allowed a rapid (5 min) and efficient pre-concentration (from $10^{-8} \mathrm{~mol} \mathrm{~L}^{-1}$ to $10^{-5} \mathrm{~mol} \mathrm{~L}^{-1}$ ), detection, and quantification of the analyte of interest, proving to be an interesting analytical tool for SERS applications.
\end{abstract}

Keywords: gold nanohole array; interference lithography; pre-concentration; Raman signature; low concentration detection

\section{Highlights:}

- $\quad$ Gold nanoholes array substrate (AuNHAS) prepared by interference lithography is a reproducible substrate for surface-enhanced Raman scattering (SERS) analysis.

- $\quad$ AuNHAS was applied as SERS substrate to detect low concentration of p-MBA.

- $\quad$ Single-drop micro-extraction (SDME) is a pre-concentration technique for low concentration analyte.

- $\quad$ Coupling SDME with SERS made possible the detection of para-mercaptobenzoic acid (p-MBA) at nanomolar concentration.

\section{Introduction}

Surface-enhanced Raman scattering (SERS) has been widely applied in chemistry, environmental analyses, material science, and biosensors, among others, as an extremely sensitive and fast technique for analytical applications, including for single-molecule detection [1-3]. The Raman scattering enhancement mechanism is extremely surface dependent, and the success of SERS depends on several factors: Most importantly, the molecule Raman cross section, its surface affinity, and the 
plasmonic activity of the nanostructures used as substrate [1,2]. Thiophenol-based molecules, as para-mercaptobenzoic acid (p-MBA), present a strong binding affinity of the thiolate group with gold for chemical attachment on the substrate surface, as well as a strong Raman signal of the benzene ring; for these reasons, they are often used as probing molecules [4].

Moreover, several nanofabrication strategies have been explored for the fabrication of SERS substrates with enhanced electric fields (hot spots), and three main classes of SERS substrates have been developed: Metallic rough surfaces, colloidal nanoparticles, and periodic nanostructures [5]. A reproducible substrate is required to obtain an excellent homogeneity of hot spots, which means high reproducibility of the SERS signal and low interference from the substrate background $[5,6]$. Gold nanohole array substrates (AuNHAS) are very attractive for this purpose, since they are periodically structured. Gold nanohole arrays of different shapes, sizes, and periodicities have been fabricated by electron beam lithography (EBL) or focused ion beam (FIB), among other techniques, and successfully applied as substrates to monitor rhodamine 6G (Rh6G) dyes and other molecules $[7,8]$. Interference lithography (IL) was employed here as an alternative fabrication method that provides a convenient approach to generate a reproducible large area AuNHAS (1-inch squared) at lower costs in respect to common serial techniques, such as FIB and EBL [9]. In a general manner, for very low concentration analysis, i.e., below $10^{-6} \mathrm{~mol} \mathrm{~L}^{-1}$, many nanostructured materials may not be sensitive enough for an efficient detection. To overcome this, a pre-concentration technique, such as the single-drop microextraction (SDME), can be introduced as an analytical strategy to improve low concentration detection.

SDME is a liquid-phase microextraction technique, where a micro drop of an organic solvent is used to transfer a dissolved substance from one liquid phase to another liquid phase (immiscible or partially miscible) in contact with it [10]. SDME has become more popular than other microextraction techniques because it is simple, cost-effective, easy to operate, and almost solvent-free [10]. The technique has been employed successfully for trace analyses in environmental, food, and sensing applications [11-13] and it has also been coupled with various analytical techniques, such as gas chromatography (GC), high-pressure liquid chromatography (HPLC), and capillary electrophoresis-mass spectrometry (CE-MS), among others [14-16]. In most cases, couplings were made in off-line mode, except for the report of Kim et al. [16], where the authors showed a direct coupling of SDME to capillary electrophoresis-mass spectrometry. On the other hand, there are no reports, to our knowledge, about coupling of SDME with SERS.

In this context, we proposed the application of a single-drop microextraction technique as a pretreatment step before SERS analysis of low concentration analytes. For this purpose, p-MBA was used as a Raman probe molecule and gold nanohole array was used as SERS substrate. The off-line coupling of SDME with SERS was applied only when direct p-MBA detection was no longer possible.

\section{Materials and Methods}

\subsection{Preparation of Gold Nanohole Array Substrates}

The gold nanohole array substrates (AuNHAS), with period $\Lambda=500 \mathrm{~nm}$, were fabricated using a combination of two-beam interference lithography (IL) and a lift-off step. Details of the experimental process can be found in Menezes et al. [9]. Briefly: One portion of the wavefront of a spatially filtered laser beam $(458 \mathrm{~nm})$ impinged directly incident on the substrate surface, while the other portion was reflected by a mirror toward the sample. The interference pattern was generated by the superimposition of these two beams. The sample was coated with a positive 500-nm thick photosensitive material. The photoresist layer was exposed twice to the same interference fringe pattern, with a $90^{\circ}$ rotation between exposures, to produce the two-dimensional template. After the exposures, with a dose of $200 \mathrm{~mJ} / \mathrm{cm}^{2}$ each, the photoresist was developed for $50 \mathrm{~s}$ with the Microposit AZ 351 developer diluted 1:3 in water. The photoresist template was coated with an $80-\mathrm{nm}$ gold film by e-beam evaporation, and then the photoresist was removed using acetone. The AuNHAS were characterized by FEG (field 
emission gun microscope, model Inspect) with $20 \mathrm{kV}$ of acceleration voltage, and energy-dispersive X-ray spectroscopy (EDS) for elemental analysis; the sample presented a homogeneous gold surface and periodic holes over an area of $2 \mathrm{~cm}^{2}$, as can be seen in Figure 1a (showing an actual total area of $\sim 10 \mathrm{\mu m}^{2}$ ). Figure $1 \mathrm{~b}$ reports the transmission spectra of the AuNHAS, showing the sharp plasmonic peak with resonance at $615 \mathrm{~nm}$ for the $(1,1)$ order.
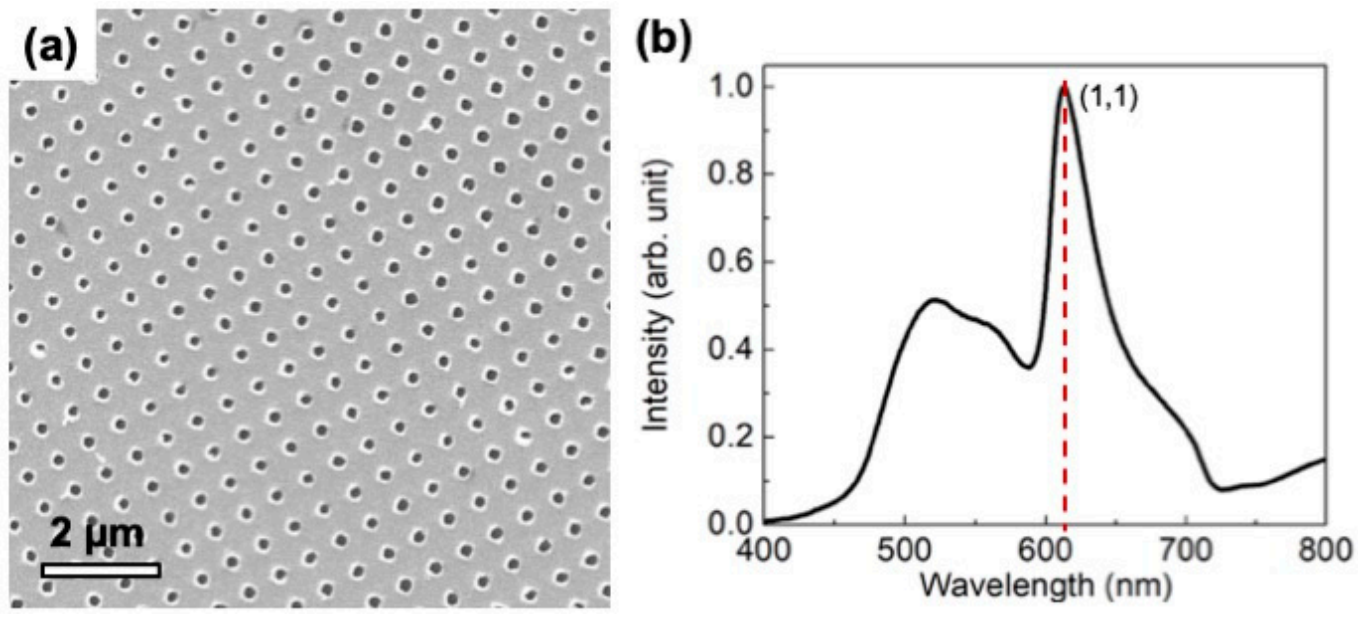

Figure 1. (a) SEM image and (b) transmission spectra of a gold nanohole array fabricated by interference lithography (IL) with a period of $\Lambda=500 \mathrm{~nm}$. The plasmonic peak of resonance order $(1,1)$ presents a maximum at $615 \mathrm{~nm}$.

\subsection{SERS Measurements}

SERS spectra were acquired using a confocal Jobin-Yvon T64000 Raman spectrometer system, equipped with a liquid- $\mathrm{N}_{2}$-cooled charge-coupled device (CCD) detector. The excitation source was a He-Ne laser at $633 \mathrm{~nm}$. The laser power at the sample surface was about $2.5 \mathrm{~mW}$. The laser was focused with a 100x focal-lens objective, to obtain a diameter of $1 \mu \mathrm{m}$ at the surface, for an exposure time of $10 \mathrm{~s}$. All the measurements were done in triplicate. After that, aliquots of $25 \mu \mathrm{L}$ from each solution of p-MBA were dropped onto the surface and dried. The surface Raman mapping data was carried out using the p-MBA solution of $10^{-6} \mathrm{~mol} \mathrm{~L}^{-1}$, collecting a total of 169 SERS spectra.

\subsection{Single-Drop Microextraction of Para-Mercaptobenzoic Acid}

The experimental setup shown in Figure 2 was applied for the SDME step. A 100- $\mu$ L Hamilton microsyringe, containing $10 \mu \mathrm{L}$ of toluene, was fixed on a platform and pressed carefully from the top in order to dip its needle into the glass vessel containing $3 \mathrm{~mL}$ of p-MBA aqueous solution, $10^{-8} \mathrm{~mol} \mathrm{~L}^{-1}$. A toluene drop of $2 \mu \mathrm{L}$ was generated inside the p-MBA solution, which was kept at $22^{\circ} \mathrm{C}$ and stirred at $100 \mathrm{rpm}$ for $5 \mathrm{~min}$. After this time, the drop was collected back and dropped onto the AuNHAS. The procedure was repeated one more time, dropping the extracted solution (p-MBA in toluene) on a different area of the same AuNHAS. After solvent evaporation, the SERS spectra were collected. 


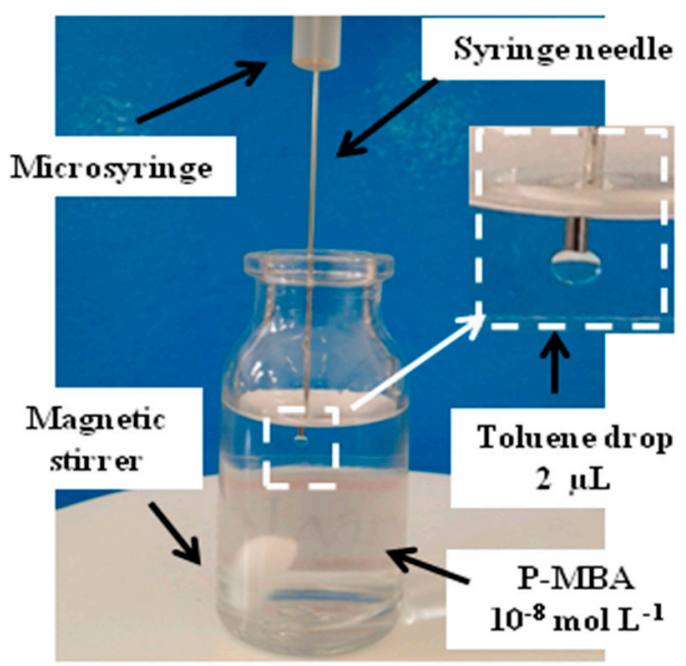

Figure 2. Single-drop microextraction (SDME) experimental setup for microextrations of para-mercaptobenzoic acid ( $p$-MBA) from a $10^{-8} \mathrm{~mol} \mathrm{~L}^{-1}$ solution. In the inset, a detailed photo of the $2 \mu \mathrm{L}$ drop extraction solvent (toluene).

\section{Results and Discussion}

Thiophenol-based molecules, such as p-MBA, are a class of compounds commonly used as Raman probe for SERS studies due to the large Raman signal of the benzene ring, offering great sensitivity and specificity [4]. Figure 3 displays one representative SERS spectra for all the p-MBA concentrations used in the study. The peak at $914 \mathrm{~cm}^{-1}$ can be related to the $\delta(\mathrm{CSH})$ bending mode, and the band at $1176 \mathrm{~cm}^{-1}$ was associated with the $\delta(\mathrm{CH})$ deformation vibration. Moreover, the intensity of the band at $1074 \mathrm{~cm}^{-1}$, assigned to the p-MBA aromatic ring breathing [4], decreased regularly when the concentration changed. For this reason, the Raman signal at $1074 \mathrm{~cm}^{-1}$ was selected as an indicator for the SERS data. Moreover, in Figure 3, the p-MBA concentration of $10^{-7} \mathrm{~mol} \mathrm{~L}^{-1}$ was considered the lowest concentration with a distinctly detected SERS signal on the fabricated AuNHAS.

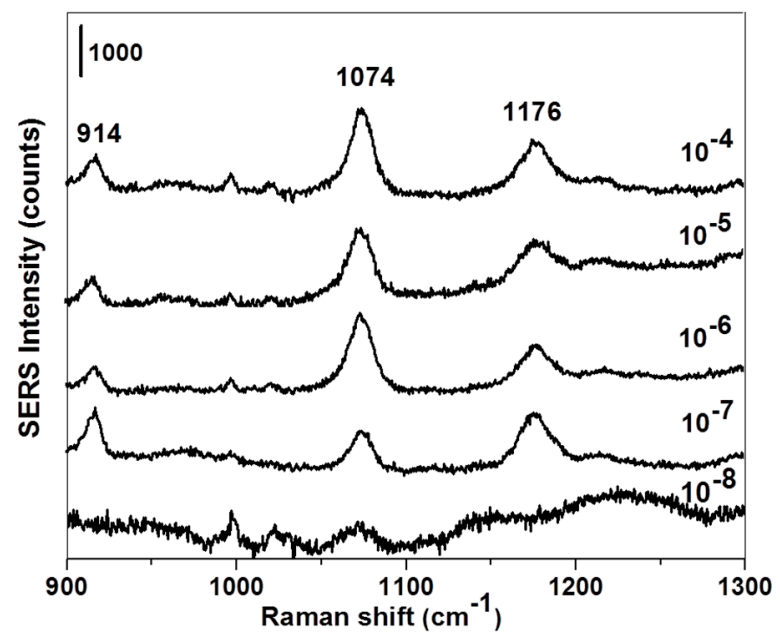

Figure 3. Representative surface-enhanced Raman scattering (SERS) spectra for p-MBA solutions at different concentrations $\left(10^{-8}-10^{-4} \mathrm{~mol} \mathrm{~L}^{-1}\right)$. Laser wavelength: $633 \mathrm{~nm}$; laser power: $2.5 \mathrm{~mW}$. The spectra were normalized and the scale bar is 1000 count. 
In order to evaluate the reproducibility of the SERS data collected on our substrate, the average SERS intensities at $1074 \mathrm{~cm}^{-1}$ for each p-MBA concentration were analyzed (Figure $4 \mathrm{a}$ ) together with the intensity distribution at $1074 \mathrm{~cm}^{-1}$ from 169 spectra (SERS mapping, Figure $4 \mathrm{~b}$ ).
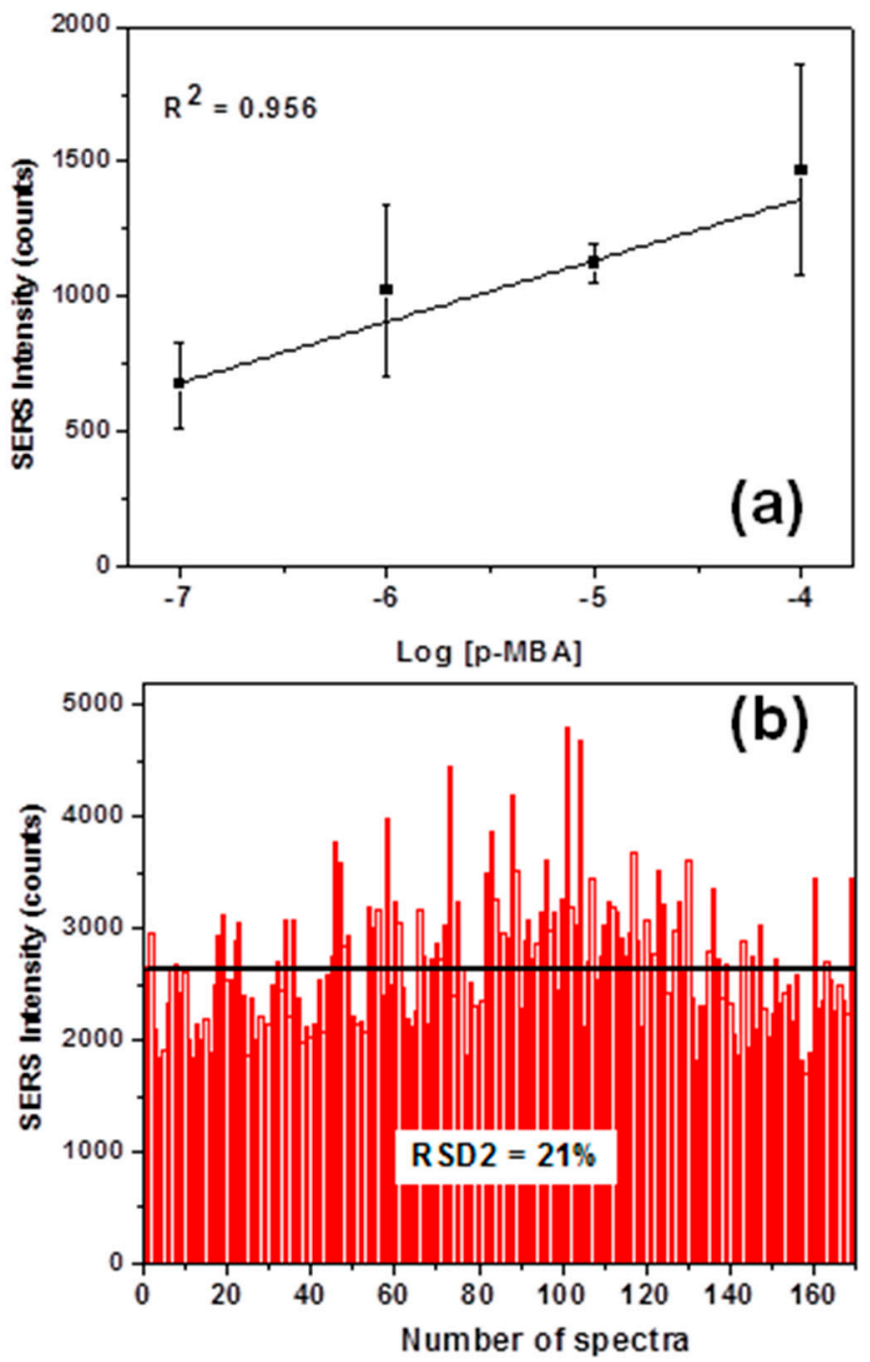

Figure 4. (a) Dependence of the average SERS intensity at $1074 \mathrm{~cm}^{-1}$, with the logarithm of the p-MBA concentrations. (b) SERS intensities distribution at $1074 \mathrm{~cm}^{-1}$ for the Raman mapping on AuNHAS of the $10^{-6} \mathrm{~mol} \mathrm{~L}^{-1} \mathrm{p}$-MBA solution. Laser wavelength: $633 \mathrm{~nm}$; laser power: $2.5 \mathrm{~mW}$.

Figure 4a demonstrates a good correlation between the average SERS intensities at $1074 \mathrm{~cm}^{-1}$ against the logarithm of p-MBA concentrations $\left(10^{-7}\right.$ to $\left.10^{-4} \mathrm{~mol} \mathrm{~L}^{-1}\right)$, leading to a coefficient of determination (R-squared) of 0.956 . This result suggests that the SERS intensities are proportional to the molecular amount of p-MBA on AuNHAS. A similar result was reported by Saleh et al. [17]; therefore, the relationship obtained can also be used as a calibration curve for future SERS quantitative analysis of p-MBA on AuNHAS. In Figure 4b, the intensity of $1074 \mathrm{~cm}^{-1}$ band from the 169 SERS spectra of the Raman mapping is plotted as a histogram: The black line in Figure 4 represents the average intensity value detected of $2671 \pm 568$ counts. The spot-to-spot variation of the band intensities was mainly caused by the differences in the localized surface plasmon resonance (LSPR), as this electromagnetic field enhancement was the major contribution toward SERS [18]. On our AuNHAS, a relative standard deviation (RSD) of $21 \%$ was achieved, indicating a good signal reproducibility, as other substrates based on LSPR reported RSD between 2030\% [18], and other good reproducible substrates presented values of slightly less than $20 \%$ [19]. 
In terms of sensitivity, the SERS detection of $p$-MBA at $10^{-8}$ mol L ${ }^{-1}$ resulted in a very weak signal (Figure 3), which cannot be clearly distinguished from the noise. To overcome this problem, the SDME technique was applied to pre-concentrate p-MBA from the $10^{-8} \mathrm{~mol} \mathrm{~L}^{-1}$ aqueous solution. In SDME, the solvent volume of extraction was an important variable for the extraction efficiency. Moreover, the amount of the analyte extracted into an organic drop was proportional to the drop size at equilibrium. In this work, $2 \mu \mathrm{L}$ of toluene was chosen as the extraction solvent volume based on the stability of the generated toluene drop in aqueous solution for $5 \mathrm{~min}$ at $22{ }^{\circ} \mathrm{C}$, from previous studies [20]. As can be observed in Figure 5, the $p$-MBA Raman signature at $1074 \mathrm{~cm}^{-1}$ was observed in the pre-concentrated SERS (SDME-SERS) spectra.

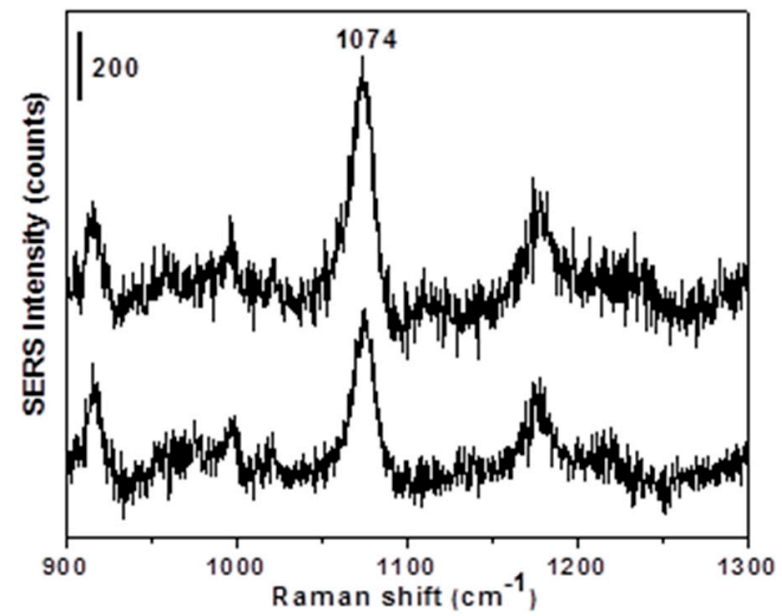

Figure 5. SERS spectra of $p$-MBA after SDME microextraction from a $10^{-8} \mathrm{~mol} \mathrm{~L}^{-1}$ solution. The SERS intensity scale bar is 200 counts. Laser wavelength: $633 \mathrm{~nm}$; laser power: $2.5 \mathrm{~mW}$.

The average SERS intensity of $1295 \pm 245$ counts was determined considering the dilution from 2 to $10 \mu \mathrm{L}$ (drop volume - volume inside the syringe) during the SDME procedure. This value is comparable with the average SERS intensity of the p-MBA solution between the concentrations $10^{-4} \mathrm{~mol} \mathrm{~L}^{-1}$ and $10^{-5} \mathrm{~mol} \mathrm{~L}^{-1}$. Moreover, the value of RSD 20\%, obtained with the combination of SDME and SERS, perfectly matches the performance of the substrate; thus, the new p-MBA concentration of $\sim 4.6 \times 10^{-5}$ mol L ${ }^{-1}$ can be extrapolated from the calibration curve (Figure $4 \mathrm{a}$ ). This result showed that SDME worked very well as a pretreatment technique, increasing the concentration of the analyte by three order of magnitude, from $10^{-8} \mathrm{~mol} \mathrm{~L}^{-1}$ to $10^{-5} \mathrm{~mol} \mathrm{~L}^{-1}$. This large improvement in the level of detection was the key issue in analytical studies and sensors, and it has been explored for many other SERS substrates using p-MBA as a probing molecule [21,22]. Although more Raman measurements should be taken to improve the calibration curve and the signal intensity variation, this work demonstrated that an easy coupling of SDME with SERS can also reach nanomolar detection levels, with the advantage of being a much simpler and faster procedure. Moreover, SDME can be easily tailored (type of solvent, temperature, and time of extraction) and applied for detection of an innumerous variety of substances, even when found in complex matrices, being an optimum alternative for detection of analyte at very low concentrations with SERS.

\section{Conclusions}

AuNHAS with a periodicity of $500 \mathrm{~nm}$ was fabricated by interference lithography and its SERS performance was investigated using $\mathrm{p}-\mathrm{MBA}$ as Raman probe molecule. Different concentrations of p-MBA were easily detected by SERS with a good reproducibility (RSD 21\%), from $10^{-4} \mathrm{~mol} \mathrm{~L}^{-1}$ up to the concentration of $10^{-7} \mathrm{~mol} \mathrm{~L}^{-1}$. To improve the analytical applicability of SERS, and lower the detection limit of this analyte, the SDME technique was successfully applied for the pre-concentration of the $10^{-8} \mathrm{~mol} \mathrm{~L}^{-1} \mathrm{p}-\mathrm{MBA}$ solution. In only five minutes, the SDME method allowed us to obtain 
a solution of analyte almost a thousand times more concentrated, from $10^{-8}$ to $\sim 4.6 \times 10^{-5} \mathrm{~mol} \mathrm{~L}^{-1}$. Moreover, the tailored choice of extraction solvent, time, and temperature can largely improve the detection of specific analytes in dense or rich matrices, greatly expanding the versatility and applicability of SDME-SERS analysis. Ultimately, for the first time, this work demonstrated that the coupling of the SDME technique with SERS analysis is a very promising method for a rapid and easy-to-apply quantitative detection of molecules at very low concentrations.

Author Contributions: Conceptualization, E.B.S. and J.W.M.; methodology, E.B.S., J.W.M. and L.F.A.; validation, E.B.S. and C.V.; formal analysis, E.B.S. and C.V.; resources, E.B.S., J.W.M. and L.F.A.; data acquisition, J.L.S.G.; writing-original draft preparation, E.B.S., C.V. and J.W.M.; writing-review and editing, C.V.

Funding: This research was funded by FAPESP, grant number 2015/16573-5.

Acknowledgments: The authors would like to thank the LMEOA/IQ/UNICAMP for the Raman facilities and the Brazilian Nanotechnology National Laboratory (LNNano, Campinas-SP, Brazil) for SEM/EDS.

Conflicts of Interest: The authors declare no conflict of interest.

\section{References}

1. Schlucker, S. Surface-Enhanced Raman Spectroscopy: Concepts and Chemical Applications. Angew. Chem. Int. Ed. 2014, 53, 4756-4795. [CrossRef] [PubMed]

2. Lane, L.A.; Qian, X.; Nie, S. SERS Nanoparticles in Medicine: From Label-Free Detection to Spectroscopic Tagging. Chem. Rev. 2015, 115, 10489-10529. [CrossRef]

3. Le Ru, E.C.; Etchegoin, P.G. Single-Molecule Surface-Enhanced Raman Spectroscopy. Annu. Rev. Phys. Chem. 2012, 63, 65-87. [CrossRef] [PubMed]

4. Sun, F.; Galvan, D.D.; Jain, P.; Yu, Q. Multi-functional, thiophenol-based surface chemistry for surfaceenhanced Raman spectroscopy. Chem. Commun. 2017, 53, 4550-4561. [CrossRef] [PubMed]

5. Fan, M.; Andrade, G.F.S.; Brolo, A.G. A review on the fabrication of substrates for surface enhanced Raman spectroscopy and their applications in analytical chemistry. Anal. Chim. Acta 2011,693, 7-25. [CrossRef] [PubMed]

6. Khlebtsov, B.N.; Khanadeev, V.A.; Panfilova, E.V.; Bratashov, D.N.; Khlebtsov, N.G. Gold nanoisland films as reproducible SERS substrates for highly sensitive detection of fungicides. ACS Appl. Mater. Interfaces 2015, 7, 6518-6529. [CrossRef] [PubMed]

7. Das, G.; Chirumamilla, M.; Toma, A.; Gopalakrishnan, A.; Zaccaria, R.P.; Alabastri, A.; Leoncini, M.; Fabrizio, E.D. Plasmon based biosensor for distinguishing different peptides mutation states. Sci. Rep. 2013, 3, 1792-1797. [CrossRef] [PubMed]

8. Yue, W.; Wang, Z.; Yang, Y.; Chen, L.; Syed, A.; Wong, K.; Wang, X. Electron-beam lithography of gold nanostructures for surface-enhanced Raman scattering. J. Micromech. Microeng. 2012, 22, 125007-125015. [CrossRef]

9. Valsecchi, C.; Armas, L.E.G.; Menezes, J.W. Large Area Nanohole Arrays for Sensing Fabricated by Interference Lithography. Sensors 2019, 19, 2182. [CrossRef] [PubMed]

10. Alothman, Z.A.; Dawod, M.; Kim, J.; Chung, D.S. Single-drop microextraction as a powerful pretreatment tool for capillary electrophoresis: A review. Anal. Chim. Acta 2012, 739, 14-24. [CrossRef]

11. Tolessa, T.; Than, Z.; Yin, Y.; Liu, J. Single-drop gold nanoparticles for headspace microextraction and colorimetric assay of mercury (II) in environmental waters. Talanta 2018, 176, 77-84. [CrossRef] [PubMed]

12. Hashemi, M.; Habibi, A.; Jahanshahi, N. Determination of cyclamate in artificial sweeteners and beverages using headspace single-drop microextraction and gas chromatography flame-ionization detection. Food. Chem. 2011, 124, 1258-1263. [CrossRef]

13. Shahvar, A.; Saraji, M.; Shamsaei, D. Headspace single drop microextraction combined with mobile phone-based on-drop sensing for the determination of formaldehyde. Sens. Actuators B Chem. 2018, 273, 1474-1478. [CrossRef]

14. Li, Y.; Xiong, Y.; Liang, Q.; Fang, C.; Wang, C. Application of headspace single-drop microextraction coupled with gas chromatography for the determination of short-chain fatty acids in RuO4 oxidation products of asphaltenes. J. Chromatogr. A 2010, 1217, 3561-3566. [CrossRef] [PubMed] 
15. Alahyari, E.; Setareh, M.; Shekari, A.; Roozbehani, G.; Soltaninejad, K. Analysis of opioids in postmortem urine samples by dispersive liquid-liquid microextraction and high performance liquid chromatography with photo diode array detection. Egypt. J. Forensic Sci. 2018, 8, 13-19. [CrossRef]

16. Kim, J.; Choi, K.; Chung, D.S. In-line coupling of single-drop microextraction with capillary electrophoresis-mass spectrometry. Anal. Bioanal. Chem. 2015, 407, 8745-8752. [CrossRef]

17. Saleh, T.A.; Al-Shalalfeh, M.M.; Al-Saadi, A.A. Graphene Dendrimer-stabilized silver nanoparticles for detection of methimazole using Surface- enhanced Raman scattering with computational assignment. Sci. Rep. 2016, 6, 32185-32196. [CrossRef]

18. Quilis, N.G.; Lequeux, M.; Venugopalan, P.; Khan, I.; Knoll, W.; Boujday, S.; de la Chapelle, M.L.; Dostalek, J. Tunable laser interference lithography preparation of plasmonic nanoparticle arrays tailored for SERS. Nanoscale 2018, 10, 10268-10272. [CrossRef]

19. Wu, W.; Liu, L.; Dai, Z.; Liu, J.; Yang, S.; Zhou, L.; Xiao, X.; Jiang, C.; Roy, V.A. Low-Cost, Disposable, Flexible and Highly Reproducible Screen Printed SERS Substrates for the Detection of Various Chemicals. Sci. Rep. 2015, 5, 10208-10217. [CrossRef]

20. Jain, A.; Verma, K.K. Recent advances in applications of single-drop microextraction: A review. Anal. Chim. Acta 2011, 706, 37-65. [CrossRef]

21. Smith, G.; Girardon, J.-S.; Paul, J.-F.; Berrier, E. Dynamics of a plasmon-activated p-mercaptobenzoic acid layer deposited over Au nanoparticles using time-resolved SERS. Phys. Chem. Chem. Phys. 2016, 18, 19567-19573. [CrossRef] [PubMed]

22. Kong, C.; Lv, J.; Sun, S.; Song, X.; Yang, Z. Copper-templated synthesis of gold microcages for sensitive surface-enhanced Raman scattering activity. RSC Adv. 2014, 4, 27074. [CrossRef]

(C) 2019 by the authors. Licensee MDPI, Basel, Switzerland. This article is an open access article distributed under the terms and conditions of the Creative Commons Attribution (CC BY) license (http://creativecommons.org/licenses/by/4.0/). 